\title{
El género Psittacanthus (Loranthaceae) en Veracruz, México
}

\section{The genus Psittacanthus (Loranthaceae) in Veracruz, Mexico}

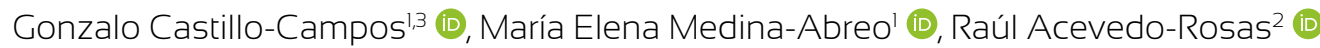

1 Instituto de Ecología, A.C., Red de Biodiversidad y Sistemática, carretera antigua a Coatepec Núm. 351, El Haya, 91070 Xalapa, Veracruz, México.

2 Universidad de Guadalajara, Departamento de Geografía y Ordenación Territorial, Guanajuato Núm. 1045, Col. Alcalde Barranquitas, 44260 Guadalajara, Jalisco, México

3 Autor para la correspondencia: gonzalo.castillo@inecol.mx

Recibido: 10 de agosto de 2017. Revisado: 14 de septiembre de 2017 Aceptado: 5 de diciembre de 2017 Primero en línea: 29 de mayo de 2018. Publicado: 1 de julio de 2018.

\section{Citar como:}

Castillo-Campos, G., M. E. Medina Abreo y R. Acevedo-Rosas. 2018. El género Psittacanthus (Loranthaceae) en Veracruz, México. Acta Botanica Mexicana 124: 19-33. DOI: 10.21829/abml24.2018.1278

DOI:

10.21829/abml24.2018.1278

\section{Resumen:}

Antecedentes y Objetivos: El género Psittacanthus es el más grande de la familia Loranthaceae, habita en zonas templadas y tropicales del Nuevo Mundo, con un amplio grupo de hospederos. El objetivo de este trabajo fue determinar las especies presentes en el estado de Veracruz, México.

Métodos: Se revisaron los especímenes de Psittacanthus colectados en Veracruz y depositados en los herbarios ENCB, MEXU y XAL. La determinación de los mismos se realizó utilizando las claves de diferentes tratamientos taxonómicos y floras, con el apoyo de colectas y observación de las especies en campo.

Resultados clave: Se lograron identificar las características morfológicas que permiten diferenciar a las cuatro especies de Psittacanthus distribuidas en Veracruz. Con esto, se resuelve la confusión que se tenía para distinguir a los taxa de este género en el estado. Además, se presenta una clave para diferenciarlos, una descripción, fotografías y mapas de distribución de cada uno de ellos.

Conclusiones: Con este trabajo se lograron esclarecer las confusiones entre las especies del género Psittacanthus que se distribuyen en Veracruz, excluyendo así mismo a P. americanus que otros autores han citado para el estado.

Palabras clave: arbustos, hemiparásitos, hospederos, muérdagos, perturbación, plaga.

\section{ABSTRACT:}

Background and Aims: The genus Psittacanthus is the largest of the family Loranthaceae, inhabiting temperate and tropical zones of the New World, with a large group of hosts. The objective of this study was to determine the species present in the state of Veracruz, Mexico.

Methods: The specimens of Psittacanthus collected in Veracruz and deposited in the herbaria ENCB, MEXU and XAL were reviewed. These were identified using keys of taxonomic treatments and floras, supported by collections and observation of the species in the field.

Key results: It was possible to identify morphological characteristics that allow to differentiate the four species of Psittacanthus distributed in Veracruz. As such, the confusion that existed to distinguish the taxa of this genus in the state could be solved. In addition, a key is presented to differentiate the species, and descriptions, photographs and distribution maps of each of them are presented.

Conclusions: With this work, it was possible to clarify the confusions among the species of the genus Psittacanthus that are distributed in Veracruz, excluding P. americanus that other authors have cited for the state.

Key words: disturbance, hemiparasites, hosts, mistletoes, plague, shrubs. 


\section{INTRODUCCIÓN}

El género Psittacanthus Mart. es un grupo de plantas arbustivas hemiparásitas, que generalmente habitan en zonas templadas y tropicales con un amplio grupo de hospederos de angiospermas y gimnospermas. A diferencia de los demás muérdagos de esta familia, se distingue fácilmente por sus flores grandes, conspicuas, de color rojo, amarillo o anaranjado, sus conexiones haustoriales voluminosas sobre los árboles hospederos, y por presentar frutos grandes, los cuales carecen de endospermo (Cházaro y Oliva, 1988; Geils y Vázquez, 2002).

Es uno de los géneros más grandes de la familia Loranthaceae. Consta de 119-120 especies (Kuijt, 2009a; Cocoletzi et al., 2016), distribuidas a lo largo del Nuevo Mundo, alcanza su límite norte en la parte central de Baja California (México), con una representación menor en el Caribe (Jamaica y algunas de las Antillas Menores) y numerosas especies en elevaciones bajas y medias en Sudamérica (Bolivia y norte de Argentina).

Aunque en México ocurren cerca de 12 especies, solamente cuatro (Geils y Vázquez, 2002; Cházaro, 2006), Psittacanthus calyculatus (DC.) G. Don, P. ramiflorus (DC.) G. Don, P. rhynchanthus (Benth.) Kuijt y $P$. schiedeanus (Schltdl. \& Cham.) G. Don, se establecen en Veracruz. Todas son hemiparásitas de árboles y arbustos y causan serios daños en algunas localidades (Geils y Vázquez, 2002). Se encuentran desde el nivel del mar hasta $2500 \mathrm{~m}$ de altitud. Se desarrollan en una gran diversidad de hospederos, incluyendo árboles y arbustos, donde para algunos como Liquidambar styraciflua $\mathrm{L}$. se han convertido en una plaga.

Es muy difícil diferenciar las especies de Psittacanthus entre sí, tanto en el campo como en las colecciones de los herbarios, si no se cuenta con estructuras reproductivas; incluso para aquellas que comparten el mismo hospedero, como ocurre frecuentemente con $P$. schiedeanus y $P$. rhynchanthus, que son los más comunes en los ambientes perturbados de las zonas tropicales del estado.

La familia Loranthaceae, a la que pertenece el género, aún no se ha revisado taxonómicamente para Vera- cruz. Sin embargo, existe la necesidad de tener un conocimiento más claro sobre los muérdagos, ya que por la perturbación que causan se están convirtiendo en una plaga principalmente para las especies arbóreas relictuales o para las que se utilizan como cercos vivos que delimitan los potreros. Por esta razón se decidió hacer este trabajo, colectando y revisando a detalle los ejemplares de herbario y las nuevas colectas.

\section{Materiales y Métodos}

Se revisaron los ejemplares de las colecciones del género Psittacanthus depositadas en los herbarios del Instituto de Ecología, A.C. (XAL), del Instituto de Biología de la Universidad Nacional Autónoma de México (MEXU) y de la Escuela Nacional de Ciencias Biológicas del Instituto Politécnico Nacional (ENCB). Los especímenes fueron corroborados desde el punto de vista morfológico, utilizando un estereomicroscopio (Stemi 2000-C, Carl Zeiss de México, S.A. de C.V., Cd. Mx., México) para evaluar sus caracteres taxonómicos, con los cuales se elaboró una clave de identificación, un cuadro sintético, y la descripción de las características morfológicas de cada especie.

También se hicieron recorridos de campo en las distintas localidades ya registradas para colectar y fotografiar in situ (cámara Canon Power Shot SX270HS, North Carolina, EUA) los especímenes con flores y frutos para diferenciarlos claramente.

Se consultaron los ejemplares escaneados de los herbarios FR (Senckenberg Research Institute), GOET (Herbarium Göttingen), K (Botanic Gardens Kew), MO (Missouri Botanical Garden), P (Field Museum of Natural History), localizados en la página electrónica JSTOR Global Plants (JSTOR, 2017) y los disponibles en la página electrónica tropicos.org (TROPICOS, 2017).

Se revisó la literatura de la Flora de Guatemala (Standley y Steyermark, 1946), Flora de Panamá (Toledo, 1960) y Flora Mesoamericana (Kuijt, 2009b).

Los mapas de distribución de los sitios de colecta se realizaron utilizando el Programa ArcGis 10.2.2 (ESRI, 2014). 


\section{Resultados}

\section{Taxonomía}

Psittacanthus Mart., Flora 13: 106. 1830. TIPO: Loranthus americanus L., Sp. Pl. 331. 1753.

Arbustos hemiparásitos, hermafroditas, sin raíces aéreas; tallos erectos, generalmente angulosos, nodos engrosados, lisos, generalmente articulados; hojas pecioladas o sésiles, opuestas, subopuestas o verticiladas, lámina coriácea o papirácea, variable en tamaño y forma; inflorescencias axilares o terminales, racemosas, umbeliformes; flores bisexuales, más de $5 \mathrm{~cm}$ de largo, pediceladas o sésiles, en díadas o tríadas, subtendidas por una bráctea, generalmente foliosa, más de $1 \mathrm{~cm}$ de largo, calículo bien diferenciado, pequeño, lóbulos de la corola 6 , estambres 6 , adnados a los pétalos, anteras dorsifijas; fruto una baya, ovoide o elipsoide; semillas sin endospermo.

En Veracruz, Psittacanthus schiedeanus y P. rhynchanthus se distribuyen principalmente en la zona tropical y subtropical de la mayor parte del estado. Psittacanthus ramiflorus y $P$. calyculatus tienen una distribución muy restringida en el sur y norte de Veracruz, en altitudes variables de 100-1500 m. En el sur del estado P. ramiflorus se encuentra en selva alta perennifolia y en bosque mesófilo de montaña, y en el norte $P$. calyculatus en el matorral crassicaule.

\section{Clave para determinar las especies del género Psittacanthus en el estado de Veracruz}

1a. Inflorescencias en díadas, no terminales

$$
\text { P. ramiflorus (DC.) G. Don }
$$

1b. Inflorescencias en tríadas, terminales 2

2a. Botones conspicuamente curvados en el ápice, rojos P. rhynchanthus (Benth.) Kuijt

2b. Botones rectos en el ápice, anaranjados 3

3a. Hojas generalmente hasta $9.5 \mathrm{~cm}$ de longitud, $4.7 \mathrm{~cm}$ de ancho, comúnmente ovadas, ocasionalmente asimétricamente ovadas; botones de menos de $6 \mathrm{~cm}$ de longitud; cotiledones 3-5

P. calyculatus (DC.) G. Don 3b. Hojas hasta $20 \mathrm{~cm}$ de longitud, hasta $8 \mathrm{~cm}$ de ancho, generalmente asimétricamente ovadas a falcadas; botones 6-8 cm de longitud; cotiledones 6-12

\section{P. schiedeanus (Schltdl. \& Cham.) G. Don}

Psittacanthus calyculatus (DC.) G. Don, Ge. Hist. 33: 415. 1834. Figs. 1A, B.

$=$ Loranthus calyculatus DC., Coll. Mem. VI. Loranthacées XXX. 1830.

$=$ L. karwinskianus Schult. \& Schult. f., Syst. Veg. 7: 1641. 1830.

$\equiv$ P. karwinskianus (Schult. \& Schult. f.) Eichler in Mart., Fl. Bras. 5(2): 26. 1868. TIPO: MÉXICO. Sultepec, Karwinski s.n. (holotipo: P!, isotipo: M).

= Chatinia calyculata (DC.) Tiegh., Bull. Soc. Bot. France 42: 351. 1895. TIPO: MÉXICO. Morelos, Cuernavaca, 1828, Berlandier 1150 (holotipo: G-DC!, isotipo: P!).

Nombre común: injerto.

Arbustos perennes, glabros, $1.3 \mathrm{~m}$ de alto, entrenudos $1-8.5 \mathrm{~cm}$ de largo, normalmente cuadrangulares, al menos cuando jóvenes, comúnmente con ángulo de los nudos redondeados, nudos no prominentes; hojas opuestas, láminas falcadas a ampliamente ovadas, 2.5-9.5(14.7) $\mathrm{cm}$ de largo, $1.8-4.7 \mathrm{~cm}$ de ancho, coriáceas, ápice redondeado, algunas veces atenuado, subsésiles o con peciolo de hasta $3 \mathrm{~mm}$ de largo, plano, venación pinnada, 5 pares de nervios secundarios, visibles en el haz, prominentes en el envés; inflorescencias terminales y subterminales con racimos de tríadas, las axilares a menudo en subumbelas de tríadas, dos o más tríadas distales, pedúnculo de la inflorescencia 10-53 $\mathrm{mm}$ de largo, pedúnculo de la tríada 9-15 mm de largo, 3-7 mm de ancho, tríadas inferiores frecuentemente con una bráctea foliácea, $2.6-4 \mathrm{~cm}$ de largo, 1.1-1.6 cm de ancho, pedicelo floral color verde, (6-)9-15 $\mathrm{mm}$ de largo, $3 \mathrm{~mm}$ de ancho, cúpula 4-5 $\mathrm{mm}$ de ancho, envolviendo 1/4 del ovario; flores en botones o antes de la antesis color anaranjado, amarillentas, escarlata brillante o ligeramente rosado, $3-5.5 \mathrm{~cm}$ de largo, rectas o 


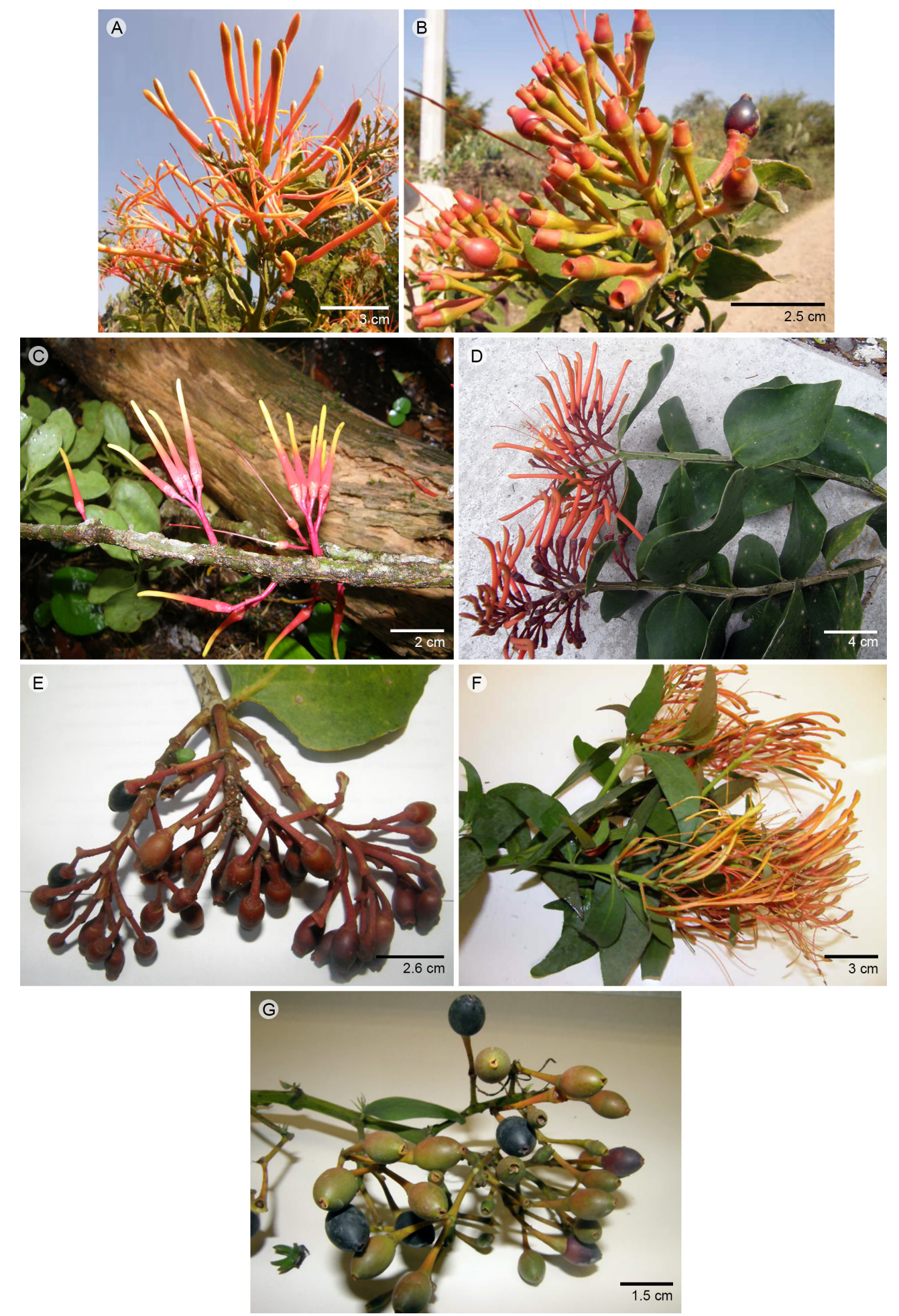

Figura 1: Caracteres diferenciales de las cuatro especies presentes en Veracruz: A, B. Psittacanthus calyculatus (DC.) G. Don, rama con inflorescencias y frutos maduros e inmaduros; pedúnculos de las flores y frutos color verde-amarillentos, flores rectas y gruesas (que difieren de las de P. schiedeanus); C. Psittacanthus ramiflorus (DC.) G. Don, rama con flores caulinares, difiere notablemente de las otras tres especies por presentar inflorescencia caulinar en díadas y flores más pequeñas de color rojo-amarillo; D, E. Psittacanthus rhynchanthus (Benth.) Kuijt, se distingue de las otras dos especies (P. calyculatus y P. schiedeanus) por presentar la rama con inflorescencia e infrutescencia terminal color rojo, frutos color rojo y negros (al madurar); el ápice de los botones curvados, agudos y las ramas articuladas; F, G. Psittacanthus schiedeanus (Schltdl. \& Cham.) G. Don, rama con inflorescencia terminal, frutos color verde y negros (al madurar), flores curvadas y delgadas con el ápice engrosado y los pedúnculos verdes. Fotografías de Gonzalo Castillo-Campos. 
escasamente inclinadas distalmente, $0.3-0.4 \mathrm{~cm}$ de grueso basalmente, estrechas en la mitad, ligeramente expandidas hasta $1 \mathrm{~cm}$ de largo, usualmente redondeadas en la punta (o puntiagudas cuando secas); calículo ligeramente dentado; pétalos color anaranjado, amarillentos, escarlata brillante o ligeramente rosado, isomórficos, $5.5 \mathrm{~cm}$ de largo, lígula basal ausente; estambres dimórficos, filamentos color anaranjado, $2.5-5 \mathrm{~cm}$ de largo, anteras $4 \mathrm{~mm}$ de largo, ligeramente septadas, redondeadas en ambos extremos, las dos series ligeramente sobrepuestas; ovario $5 \mathrm{~mm}$ de largo, $3 \mathrm{~mm}$ de ancho, estilo color anaranjado, recto, $2.7-4.1 \mathrm{~cm}$ de largo, $0.04-0.05 \mathrm{~cm}$ de ancho, liso, estigma finamente papilado; frutos numerosos, frecuentemente en tríadas, color rojo, negro-azulados al madurar, ovoides, 10-15 mm de largo, 8-10 mm de diámetro, pedicelos color verde-amarillento, anaranjados en frutos maduros, $1.3-2.2 \mathrm{~cm}$ de largo, $0.2-0.35 \mathrm{~cm}$ de ancho, calículo conspicuo; embrión con 3-5 cotiledones.

Distribución, hábitat y estado de conservación: es una especie endémica de México; se distribuye en los estados de Aguascalientes, Chiapas, Colima, Estado de México, Guerrero, Jalisco, Michoacán, Morelos, Nayarit, Oaxaca, Puebla, Querétaro, Sinaloa y Veracruz. En el estado de Veracruz crece al norte, en el municipio Huayacocotla, en matorral crassicaule, entre 1960 y $2150 \mathrm{~m}$ de altitud (Fig. 2). De acuerdo con la Norma Oficial Mexicana de especies en riesgo (SEMARNAT, 2010) y la Lista Roja de

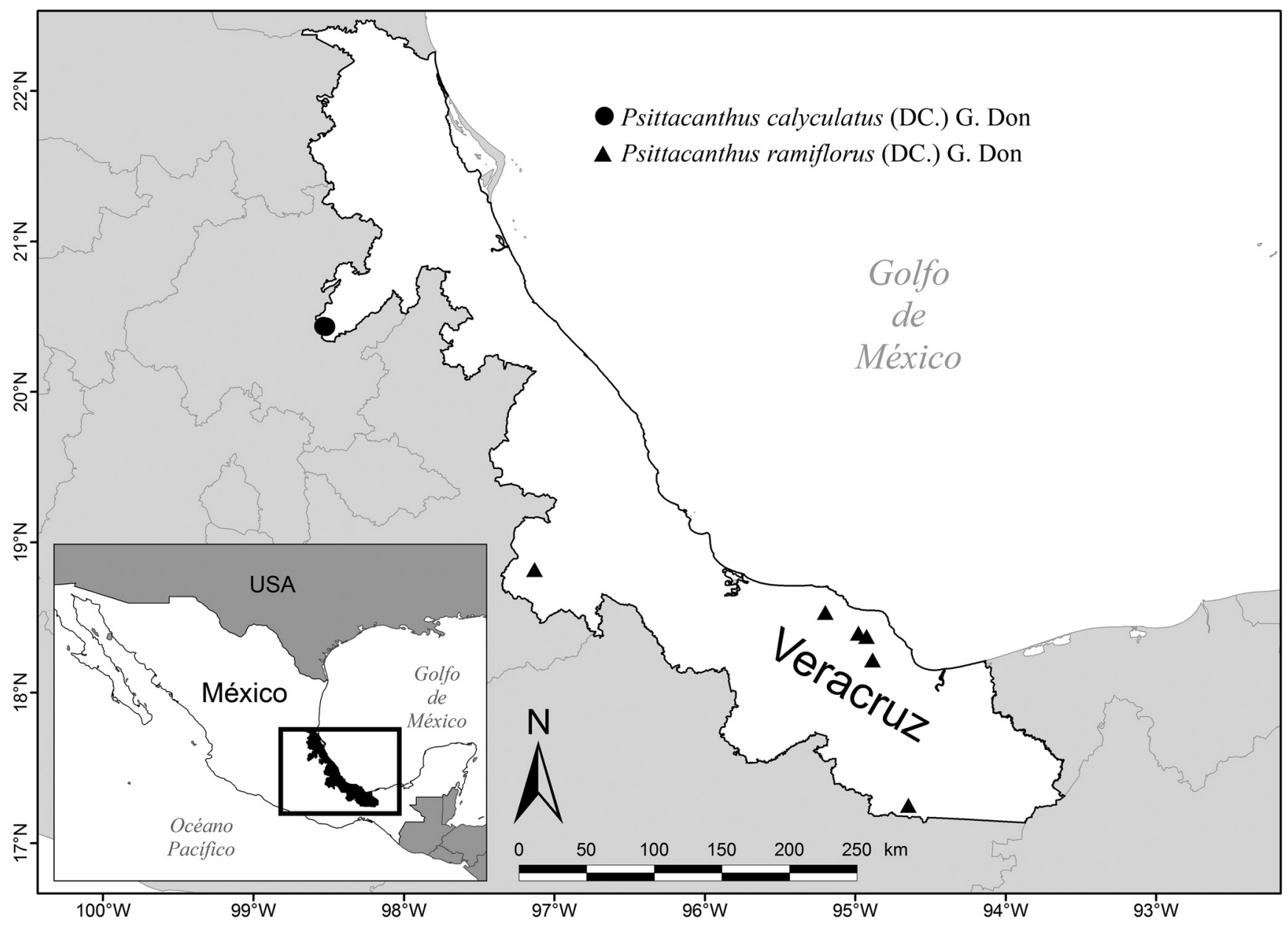

Figura 2: Distribución de Psittacanthus calyculatus (DC.) G. Don y P. ramiflorus (DC.) G. Don. 
la Unión Internacional para la Conservación de la Naturaleza (IUCN, 2017), así como lo observado en el campo, el estado de conservación de Psittacanthus calyculatus se considera como no amenazada. Florece y fructifica de septiembre a octubre y parasita árboles de Acacia Mill.

Ejemplares examinados: MÉXICO. Veracruz, municipio Huayacocotla, al N de Santiago, G. CastilloCampos y M. Escamilla B. 28501 (XAL); El Zapote, G.
Castillo-Campos y M. Escamilla B. 28600 (XAL), 28602 (XAL), 28603 (XAL), 28604 (XAL).

Comentarios taxonómicos: Psittacanthus calyculatus ha sido frecuentemente confundido en Veracruz con $P$. rhynchanthus; sin embargo, esta última especie se puede distinguir porque tiene hojas falcadas más grandes, pedicelos, flores, frutos y en general la inflorescencia de color rojo y los ejes de la infrutescencia articulados (Cuadro 1).

Cuadro 1: Caracteres morfológicos de las cuatro especies de Psittacanthus Mart. distribuidas en Veracruz.

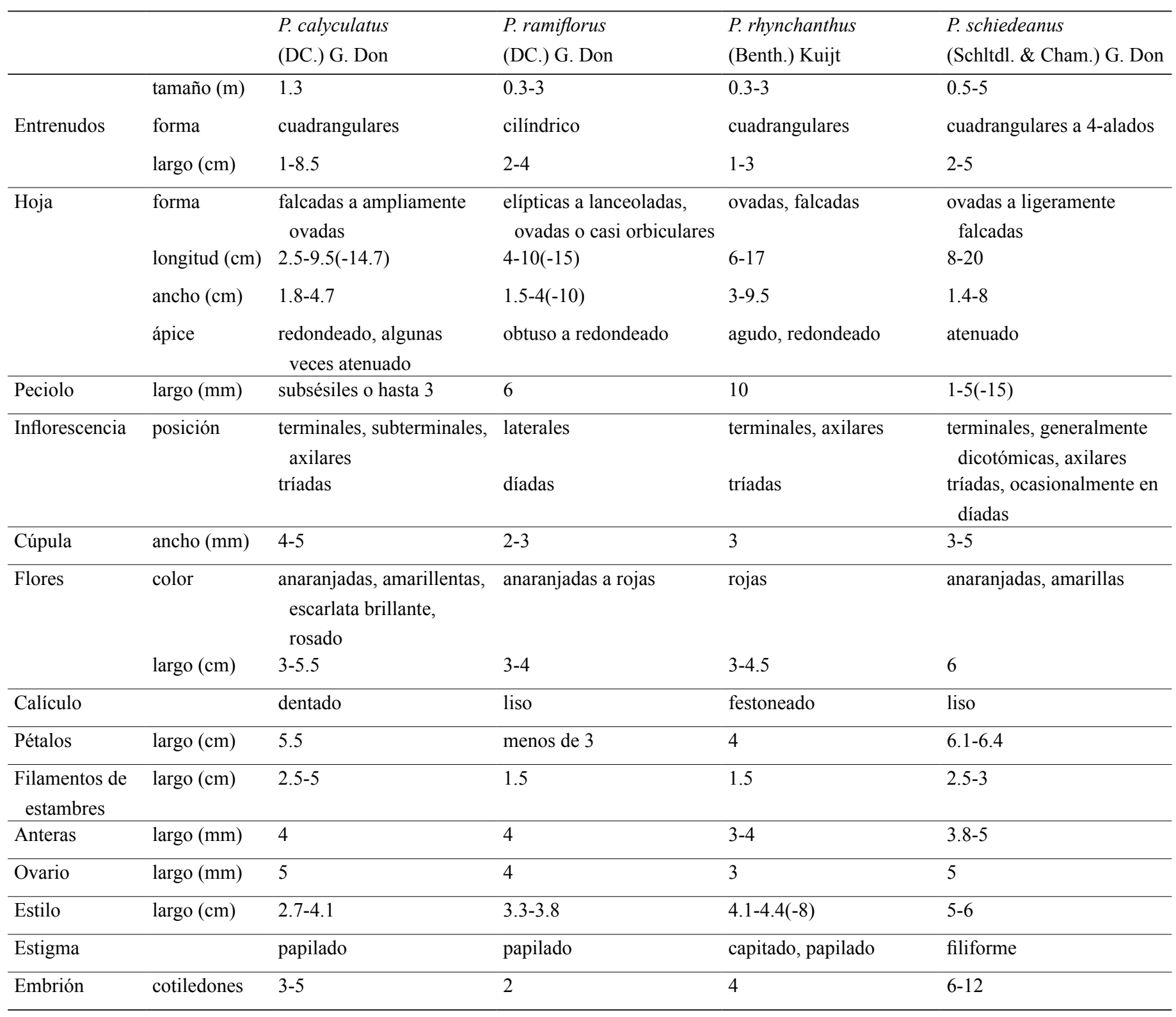


Psittacanthus ramiflorus (DC.) G. Don, Gen. Hist. 3: 415. 1834.

=Loranthus ramiflorus DC., Prodr. 4: 308. 1830. TIPO: Ilustración de L. ramiflorus, en A. L. P. P. de Candolle, Caiques des dessins de la flore du Mexique, de Mociño et Sessé, Geneve, 1874. Fig. 1C.

$\equiv$ P. allenii Woodson \& Schery, Ann. Missouri Bot. Gard. 27: 309. 1940. TIPO: PANAMÁ. Coclé, alrededor de El Valle, 600-1000 m, 8.XII.1938, P. H. Allen 1223 (holotipo: $\mathrm{MO}$ !, isotipo: $\mathrm{F}$ !).

$\equiv$ P. lateriflorus Woodson \& Schery, Ann. Missouri Bot. Gard. 27: 309. 1940. TIPO: PANAMÁ. Coclé, alrededor del Valle de Antón, 600 m, 17.IX.1939, P. H. Allen 1979 (holotipo: MO!, isotipos: F!, NY!, US!).

$\equiv$ P. scheryi Woodson, Ann. Missouri Bot. Gard. 28: 426. 1941. TIPO: PANAMÁ. Chiriqui, alrededor de Bajo Mono y Quebrada Chiquero, 18.VII.1940, R. E. Woodson y R. W. Schery 581 (holotipo: MO!, isotipo: US!).

Nombres comunes: no registrados.

Arbustos o hierbas perennes, glabros, 0.3-3 m de alto, entrenudos 2-4 $\mathrm{cm}$ de largo, cilíndricos, generalmente con lenticelas prominentes, nudos no prominentes; hojas irregularmente pareadas, frecuentemente con 4-10 pares, alternas o verticiladas, láminas 4-10(-15) cm de largo, 1.5-4(-10) $\mathrm{cm}$ de ancho, ligeramente elípticas a lanceoladas, ovadas o casi orbiculares, coriáceas, ápice obtuso a redondeado, base obtusa a cuneada, peciolo 6 mm de largo, venación pinnada, inconspícua, excepto el nervio central; inflorescencias laterales en los tallos de las ramas, frecuentemente agrupadas en díadas, pedúnculo de la inflorescencia 2-4 $\mathrm{mm}$ de largo, color rojo, pedicelo floral 4-6 $\mathrm{mm}$ de largo, color rojo, cúpula 2-3 $\mathrm{mm}$ de ancho, color rosado; flores en botones o antes de la antesis color anaranjado a rojo, 3-4 cm de largo, dilatadas en la parte inferior, la porción superior ligeramente inclinada, ápice redondeado, color rojo brillante en la parte inferior; calículo liso; pétalos color anaranjado a rojo, isomórficos, menos de $3 \mathrm{~cm}$ de largo, lígulas basales pequeñas, decurrentes, glabras; estambres irregularmente dimórficos, fasciculados alrededor del estilo, rectos en la antesis, filamentos color morado, $1.5 \mathrm{~cm}$ de largo, anteras $4 \mathrm{~mm}$ de largo; ovario $4 \mathrm{~mm}$ de largo, $3 \mathrm{~mm}$ de ancho, estilo color anaranjado, recto, 3.3-3.8 $\mathrm{cm}$ de largo, 0.03-0.05 $\mathrm{cm}$ de ancho, liso, estigma ligeramente papilado; frutos en díadas, color rojo, negros al madurar, elipsoides, cerca de $12 \mathrm{~mm}$ de largo, $8 \mathrm{~mm}$ de diámetro, pedicelos color rojo, 0.85-0.9 cm de largo, 0.1-0.15 cm de ancho, calículo inconspícuo; embrión con 2 cotiledones.

Distribución, hábitat y estado de conservación: es una especie poco frecuente, pero de amplia distribución en México (Chiapas, Guerrero, Jalisco, Nayarit y Oaxaca), Costa Rica y Panamá. En Veracruz crece entre 100 y $1500 \mathrm{~m}$ de altitud en el sur del estado (Fig. 2). Aunque es una especie escasa, no se encuentra amenazada (SEMARNAT, 2010). Generalmente se desarrolla en selva alta perennifolia, selva baja caducifolia, bosque mesófilo de montaña y bosque de Quercus. Considerando que parasita hospederos de la vegetación original, es probable que el cambio de uso del suelo ocasione que la ubiquen como amenazada. Sin embargo, en años recientes se ha encontrado parasitando árboles del género Citrus L. Florece y fructifica de abril a junio.

Ejemplares examinados: MÉXICO. Veracruz, municipio Catemaco, La Otra Opción, en el sendero interpretativo del rancho, G. Castillo-Campos et al. 28187 (XAL). Municipio Huiloapan, cerro San Cristóbal, M. Cházaro B. et al. 1412 (XAL). Municipio San Andrés Tuxtla, volcán San Martín Tuxtla, R. Cedillo T. 3172 (MEXU, XAL). Municipio Soteapan, cumbre de El Bastonal, $14 \mathrm{~km} \mathrm{SE}$ del lago Catemaco, J. H. Beaman 6143 (XAL); cima del cerro Platanillo, sierra de Santa Martha, J. I. Calzada 5123 (XAL). Municipio Uxpanapa, $4 \mathrm{~km}$ por el camino y vereda al $\mathrm{S}$ de entronque de terracería La Laguna - Sarabia con el camino al $\mathrm{N}$ a Poblado Dos (entronque que está $16.2 \mathrm{~km}$ al W de La Laguna), T. Wendt e I. Navarrete 3249 (ENCB, MEXU). 
Psittacanthus rhynchanthus (Benth.) Kuijt, Ann. Missouri Bot. Gard. 74: 529. 1987. Figs. 1D, E.

=Loranthus rhynchanthus Benth., Bot. Voy. Sulphur 102. 1845. TIPO: HONDURAS. Gulf of Fonseca, Tiger Island, Dr. Sinclair s.n. (holotipo: K!).

$\equiv$ P. chrismarii Urb., Bot. Jahrb. Syst. 24: 13. 1897. TIPO: COSTA RICA. Guanacaste, forests of Nicoya, Tonduz 13706 (neotipo: designado por Kuijt, 1987, US!, isoneotipos: CR!, GH!, K!).

EP. semiarticulatus Rizzini, Rodriguésia 30/31:142. 1956. TIPO: VENEZUELA. Guarico, Parapara, 12.IX.1927, $H$. Pittier 12532 (holotipo: VEN!, isotipos: MO!, NY!, US!).

Nombres comunes: caballera, chaya, corrigüela, hiedra, higo de chaca, secapalo y tepalcayo.

Arbustos o hierbas perennes, 0.3-3 m de alto, glabros, escasamente ramificados, entrenudos frecuentemente 1-3 cm de largo, cuadrangulares, incluso cuando viejos, color grisáceo en seco o verde amarillento, nudos no prominentes; hojas pareadas, las más pequeñas ovadas, las más largas falcadas, 6-17 cm de largo, 3-9.5 cm de ancho, ápice agudo, redondeado, base aguda, obtusa, venación impresa, ligeramente palmada, peciolo $10 \mathrm{~mm}$ de largo; inflorescencias terminales, con 2-3 pares de tríadas, en ocasiones contraídas en un espiral de tríadas, 1-varias por axila, pedúnculos color rojo, 9-12.5 $\mathrm{mm}$ de largo, $2 \mathrm{~mm}$ de ancho, expandido hacia la parte apical, pedicelos color rojo, 11-15 mm de largo, $2 \mathrm{~mm}$ de ancho, brácteas y cúpulas $3 \mathrm{~mm}$ de ancho cuando secas; flores en botones o antes de la antesis color rojo, 3-4.5 cm de largo, $1 \mathrm{~cm}$ de grueso, punta curvada hacia arriba, terminando en un ápice estrecho, puntiagudo, conspicuo, base ligeramente dilatada; calículo irregularmente festoneado; pétalos color rojo, 4 cm de largo, ligeramente dimórficos, pelos color rojo, largos sobre la superficie interna, desde la inserción del filamento hasta el ápice, lígulas basales ausentes; estambres dimórficos, filamentos $1.5 \mathrm{~cm}$ de largo, anteras 3-4 mm de largo, las dos series ligeramente sobrepuestas; ovario $3 \mathrm{~mm}$ de largo, $2.5 \mathrm{~mm}$ de ancho, estilo color anaranjado, curvado, 4.1-4.4(-8) $\mathrm{cm}$ de largo, 0.03-0.05 $\mathrm{cm}$ de ancho, liso, estigma capitado, papilado; frutos numerosos por infrutescencia, frecuentemente en díadas, ocasionalmente en mónadas, rara vez en tríadas, color rojo, púrpura a negro brillante al madurar, elipsoides, 11-15 mm de largo, 7-9 $\mathrm{mm}$ de diámetro, pedicelos $0.8-1.3 \mathrm{~cm}$ de largo, 0.1-0.2 cm de ancho, calículo prominente; embrión con 4 cotiledones.

Distribución, hábitat y estado de conservación: es una especie de amplia distribución en México (Campeche, Chiapas, Colima, Estado de México, Guerrero, Jalisco, Michoacán, Morelos, Nayarit, Oaxaca, Puebla, Quintana Roo, Tabasco, Yucatán y Zacatecas), Belice, Costa Rica, El Salvador, Guatemala, Honduras, Nicaragua, Panamá, norte de Colombia, Venezuela y Trinidad y Tobago. Crece desde el nivel del mar hasta $1400 \mathrm{~m}$ de altitud, desde el sur hasta el norte del estado de Veracruz (Fig. 3). Se presenta con mayor frecuencia en los bosques tropicales y también habita en bosque de Quercus, bosque mesófilo de montaña, bosque tropical caducifolio, bosque tropical perennifolio, bosque tropical subcaducifolio, matorral xerófilo, pastizal, vegetación acuática y subacuática; pierde su follaje en la estación seca. Es una especie que no se encuentra amenazada (SEMARNAT, 2010; IUCN, 2017). Florece y fructifica de julio a marzo. Sus hospederos son diversos, entre los que destacan los géneros Ficus L. y Bursera Jacq. ex L.

Ejemplares examinados: MÉXICO. Veracruz, municipio Actopan, estación de biología de La Mancha, $G$. Castillo-Campos 6865 (XAL); cerca de la estación biológica de La Mancha, CICOLMA, G. Castillo-Campos et al. 16405 (XAL); estación de biología de La Mancha, $H$. Oliva R. y M. Cházaro B. 23 (XAL); estación de biología de La Mancha, F. Ramírez R. 2180 (XAL); La Mancha, J. Sánchez T. 02 (XAL). Municipio Alvarado, Conejos, laguna Conejos, G. Castillo-Campos y J. Pale P. 24275 (MEXU, XAL); km 14, carretera 180 Veracruz - Alvarado, rancho 3 M, S. Olvera F. 47 (MEXU), 132 (MEXU). Municipio Apazapan, M. Cházaro B. 2557 (XAL). Mu- 


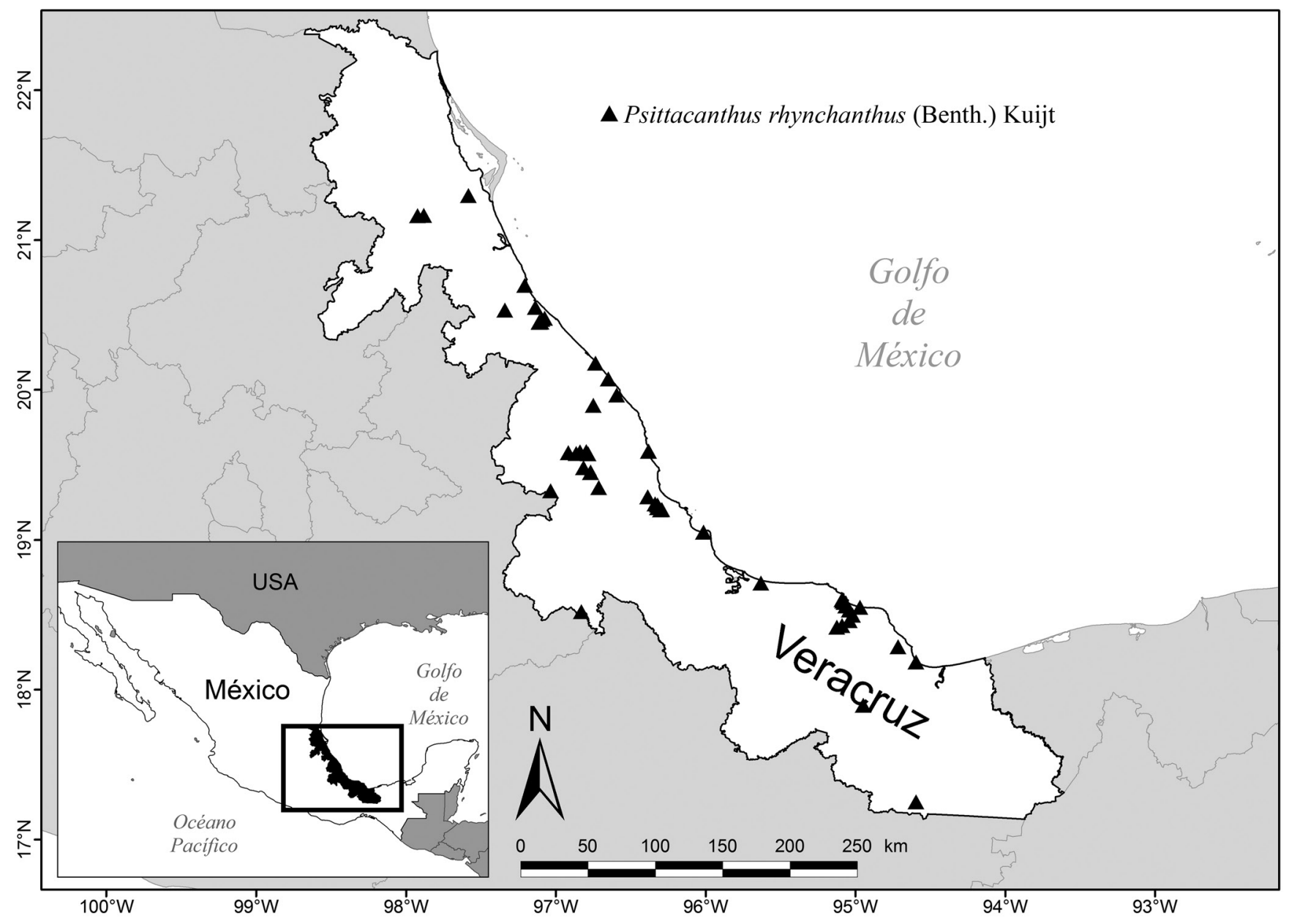

Figura 3: Distribución de Psittacanthus rhynchanthus (Benth.) Kuijt.

nicipio Catemaco, lago de Catemaco, J. I. Calzada 37 (MEXU); brecha a Calpultéotl, entrando por Panga, a través de la barra de Sontecomapan, A. Campos V. 7770 (MEXU); laguna de Sontecomapan, $20 \mathrm{~km}$ al NE del camino de terracería Catemaco - Montepío, R. Cedillo $T$. 2885 (MEXU, XAL); La Palma, T. P. Ramamoorthy 4070 (MEXU); playa Azul, laguna de Catemaco, M. Sousa 2625 (MEXU); río Coxcoapan, camino a Sontecomapan, R. Torres C. y H. Hernández 6455 (MEXU). Municipio Cazones, Barra de Cazones, 3 km, L. Monroy et al. 108 (MEXU). Municipio Coatzacoalcos, cerca de Barrillas, $A$. Lot H. 1647 (MEXU, XAL). Municipio Emiliano Zapata, El Lencero, SE, cerca de la cañada, H. Oliva R. y M. Cházaro B. 50 (XAL); Corral Falso, camino a Pinoltepec, $H$.
Oliva R. 100 (XAL); La Laja, entre Corral Falso y Pinoltepec, Puga et al. 7907 (MEXU); La Laja, $900 \mathrm{~m}$ camino Xalapa - Veracruz, $16 \mathrm{~km}$ al SE Xalapa, entre Corral Falso y Pinoltepec, M. Sousa S. y C. H. Ramos 4719 (MEXU); arroyo del Chino, $2 \mathrm{~km}$ al NE de La Balsa, F. Vázquez B. 450 (XAL). Municipio Gutiérrez Zamora, $1.5 \mathrm{~km}$ al N de Gutiérrez Zamora, sobre la carretera a Tecolutla, $G$. Castillo-Campos y M. E. Medina A. 28357 (XAL), 28358 (XAL); $1 \mathrm{~km}$ al $\mathrm{N}$ de Gutiérrez Zamora, por la carretera a Boca de Lima, G. Castillo-Campos y M. E. Medina A. 28359 (XAL); $1.5 \mathrm{~km}$ carretera Gutiérrez Zamora - Papantla, G. Castillo-Campos y M. E. Medina A. 28370 (XAL); El Ojite, $2 \mathrm{~km}$ al NW de Gutiérrez Zamora, $G$. Castillo-Campos y M. E. Medina A. 28371 (XAL); Gu- 
tiérrez Zamora, M. E. Cortés 95 (MEXU, XAL). Municipio Ixhuacán de los Reyes, Pocitos, camino a Ixhuacán de los Reyes, H. Oliva R. y M. Cházaro B. 20 (XAL). Municipio La Antigua, Vargas, camino a Loma Iguana, P. Zamora C. y M. G. Zolá B. 140 (XAL); Loma Iguana, $2 \mathrm{~km}$ al W, P. Zamora C. 573 (XAL). Municipio Naolinco, al W de Almolonga, G. Castillo-Campos et al. 17101 (XAL). Municipio Nautla, entrada al hotel Istirinchá, $G$. Castillo-Campos y M. E. Medina A. 28372 (XAL). Municipio Papantla, Poza Rica, camino a Santa Águeda, M. E. Cortés 526 (MEXU, XAL). Municipio Paso de Ovejas, E1 Hatito, camino de terracería a Caño Prieto, $1 \mathrm{~km}$ del Hatito, J. E. González H. 188 (XAL). Municipio San Andrés Tuxtla, lote 67, estación de biología tropical Los Tuxtlas, G. Ibarra M. 477 (MEXU); estación de biología tropical Los Tuxtlas, G. Ibarra M. 954 (ENCB, MEXU, XAL); N and $\mathrm{E}$ sides of laguna Encantada, $3 \mathrm{~km}$ NE of San Andrés Tuxtla, M. Nee 24760 (XAL); Laguna Escondida, $3.5 \mathrm{~km}$ NW de la estación de biología tropical Los Tuxtlas, G. A. Salazar y S. Sinaca C. 303 (MEXU); circuito de la estación de biología tropical Los Tuxtlas, G. A. Salazar 305 (MEXU); Laguna Escondida, $3 \mathrm{~km}$ al NW de la estación de biología tropical Los Tuxtlas, S. Sinaca C. y F. Chigo S. 228 (ENCB, MEXU, XAL); Laguna Escondida, 2 km al NW de la estación de biología tropical Los Tuxtlas, $S$. Sinaca C. 1577 (XAL), 1578 (MEXU). Municipio Sayula de Alemán, km 5 on hwy 185 Acayucan to Salina Cruz, $\mathrm{S}$ of autopista, C. A. Pendry y A. Reyes 933 (MEXU). Municipio Tamiahua, Tampache, camino de terracería a Hormiguero, A. Rincón G. y C. Durán E. 1272 (MEXU, XAL). Municipio Tecolutla, Boca de Lima, G. CastilloCampos y M. E. Medina A. 28364 (XAL). Municipio Tepetzintla, $2.7 \mathrm{~km} \mathrm{SE}$, along hwy 105, M. Nee 22396 (XAL); 30-35 km al S de Mata Redonda (Tampico - Tuxpan), L. I. Nevling y A. Gómez-Pompa 462 (XAL). Municipio Tezonapa, Vista Hermosa, $3 \mathrm{~km}$ al SW, R. Robles G. 1022 (MEXU, XAL). Municipio Vega de Alatorre, al $\mathrm{N}$ de Vega de Alatorre, $5 \mathrm{~km}$ hacia playa Navarro, G. Castillo-Campos y M. E. Medina A. 28374 (XAL); potrero Bougambilia, Llanos de Muchachos, Emiliano Carranza, cerca de arroyo El Baño, A. Lot H. 615 (MEXU). Munici- pio Veracruz, Nevería, carretera antigua Nacional Xalapa - Veracruz, C. Gutiérrez B. 833 (XAL); 2 km al W de Santa Fe, C. Gutiérrez B. 1226 (ENCB, MEXU, XAL); Paso San Juan, $1.5 \mathrm{~km}$ al N, V. E. Luna M. y M. G. Zolá B. 380 (XAL). Municipio Xalapa, near Xalapa, M. Cházaro B. et al. 1010 (XAL); ca. de San Antonio Paso del Toro, ca. de $15 \mathrm{~km}$ al N de Actopan, S. Galen Smith 6054 (XAL); Chiltoyac, M. G. Zolá B. 773 (MEXU, XAL). Municipio Yecuatla, El Ojite, desviación a La Colonia de Colipa, $C$. Gutiérrez B. 4099 (XAL).

Comentarios taxonómicos: Psittacanthus rhynchanthus estuvo identificado en la mayoría de los ejemplares de herbario como P. calyculatus; sin embargo, $P$. rhynchanthus se distingue claramente de $P$. calyculatus por los caracteres ya mencionados.

Psittacanthus schiedeanus (Schltdl. \& Cham.) G. Don, Gen. Hist. 3: 418. 1834.

= Loranthus schiedeanus Schltdl \& Cham., Linnaea 5: 172. 1830. TIPO: MÉXICO. Veracruz, cerca de Jalapa, Schiede y Deppe 404 (holotipo: B, destruido; isotipos: LE, M!, MO!). Figs. 1F, G.

$=$ L. kerberi E. Fourn., Bull. Soc. Bot. France 30: 185. 1883.

=L. quauchitli Sessé y Moc., PI. Nov. Hisp. 3: 47. T. 65. 1888. TIPO: MÉXICO. Type drawing (G), Sessé y Mociño s.n. 1787-1803.

$\equiv$ P. kerberi (E. Fourn.) Engl., Nat. Pflanzenfam., Nachtr. Zum II-IV. Teil, 136. 1897. TIPO: MÉXICO. Veracruz, Córdoba, 18.VIII.1882, Kerber 36 (holotipo: B, destruido, foto: F negativo 11829!, isotipos: BM!, C, F!, GH, K!, P!, US!).

Nombres comunes: corrigüela, tepalcatillo y tepalcayo.

Arbustos perennes, ramificados, glabros, 0.5-5 m de alto, entrenudos 2-5 cm de largo, 0.5-0.6 cm de ancho, cuadrangulares a estrechamente 4 -alados, a menudo 
conspicuamente planos debajo de los nudos, 0.2-0.3(-0.6) $\mathrm{cm}$ de diámetro, nudos no prominentes; hojas frecuentemente opuestas, verticiladas, ocasionalmente alternas, láminas color verde, a menudo asimétricas, ovadas a ligeramente falcadas, $8-20 \mathrm{~cm}$ de largo, $1.4-8 \mathrm{~cm}$ de ancho, ápice atenuado, base atenuada, aguda a obtusa, venación pinnada, haz color verde, nervio central impreso, envés color verde claro, nervio central ligeramente prominente, peciolo 1-5(-15) $\mathrm{mm}$ de largo, 1-2 $\mathrm{mm}$ de ancho; inflorescencias terminales, generalmente dicotómicas, 9-14 cm de largo, en su mayoría con una inflorescencia secundaria cerca de las axilas foliares, con 3-5 pares de tríadas, frecuentemente dispuestas en tríadas, ocasionalmente en díadas, pedúnculo color verde a anaranjado-amarillento, 16-24 $\mathrm{mm}$ de largo, $2 \mathrm{~mm}$ de ancho, brácteas asimétricas, agudas, ligeramente falcadas, 2.5-5.8 $\mathrm{mm}$ de largo, ápice agudo, atenuado, base atenuada, pedicelos florales anaranjados, 14-20 mm de largo, 1.8-2 mm de ancho, cúpula 3-5 $\mathrm{mm}$ de ancho; flores en botones o antes de la antesis color anaranjado, amarillo, $6 \mathrm{~cm}$ de largo; calículo color verde, 0.34-0.4 cm de largo, 0.26-0.3 cm de ancho, liso; pétalos 6 , lineares, fusionados cerca de $0.08 \mathrm{~cm}$ en la base, 6.1-6.4 cm de largo, 0.8-1 cm de ancho; estambres dimórficos, filamentos cortos, color anaranjado, $2.5-3 \mathrm{~cm}$ de largo, $0.04 \mathrm{~cm}$ de ancho, filamentos largos 3-3.4 cm de largo, $0.04 \mathrm{~cm}$ de ancho, anteras 3.8-5 $\mathrm{mm}$ de largo, 0.9$1.4 \mathrm{~mm}$ de ancho, alveoladas, las dos series sobrepuestas; ovario $5 \mathrm{~mm}$ de largo, $3.5 \mathrm{~mm}$ de ancho, estilo color anaranjado, recto, 5-6 cm de largo, 0.04-0.06 cm de ancho, liso, estigma pequeño, filiforme; frutos frecuentemente en tríadas, ocasionalmente en díadas, color verde, rojizo, negro al madurar, elipsoides, 12-15 mm de largo, 8-10 mm de diámetro, pedicelos color verde, amarillo-anaranjado cuando el fruto está maduro, $2-2.5 \mathrm{~cm}$ de largo, $0.2 \mathrm{~cm}$ de ancho, calículo prominente; embrión con 6-12 cotiledones prismáticos a lanceolados.

Distribución, hábitat y estado de conservación: Psittacanthus schiedeanus es de amplia distribución en México (Chiapas, Estado de México, Guerrero, Jalisco, Michoacán, Morelos, Nayarit, Oaxaca, Puebla, Querétaro,
San Luis Potosí y Tamaulipas), Costa Rica, El Salvador, Guatemala, Honduras y Panamá. En Veracruz se distribuye desde el nivel del mar hasta los $2000 \mathrm{~m}$ de altitud, en diferentes tipos de vegetación (bosque de Quercus, mesófilo de montaña, tropical caducifolio, tropical perennifolio y tropical subcaducifolio, vegetación acuática y subacuática) (Fig. 4). Parasita diversas especies de árboles y arbustos; entre los géneros más comunes están Bursera, Ficus, Liquidambar L., entre otros. Es una especie que no se encuentra amenazada (SEMARNAT, 2010; IUCN, 2017) y se puede convertir en plaga en árboles aislados, después de la remoción de la vegetación original. Florece $\mathrm{y}$ fructifica de junio a diciembre.

Ejemplares examinados: MÉXICO. Veracruz, municipio Actopan, Mozomboa, $2 \mathrm{~km}$ al N, camino al cerro de La Mesa, R. Acosta P. y N. Acosta B. 154 (MEXU, XAL); Raya Los Chalahuites, Sierra Manuel Díaz, $R$. Acosta P. 819 (MEXU, XAL); playa Cansa Burros, 2 km al S de La Mancha, R. Acosta P. y F. Vázquez B. 820 (XAL); estación de biología Morro de La Mancha, Brigada de dunas 405 (MEXU); $3 \mathrm{~km}$ al NE de la desviación a Otates, G. Castillo-Campos e I. Acosta R. 19372 (MEXU, XAL); estación de biología de La Mancha, $B$. Guerrero C. 2150 (MEXU, XAL); estación de biología de La Mancha, M. Keyes H. y C. Armenta C. 32 (XAL); estación de biología de La Mancha, P. Moreno-Casasola 1225 (MEXU); estación de biología de La Mancha, $R$. Ortiz-Pulido 12 (MEXU, XAL); estación de biología de La Mancha, F. Ramírez R. 2184 (XAL). Municipio Alto Lucero, $1 \mathrm{~km}$ al $\mathrm{N}$ de Santander, G. Castillo-Campos y M. E. Medina A. 28375 (XAL). Municipio Apazapan, Apazapan, $1 \mathrm{~km}$ al E, G. Castillo-Campos y A. Birke B. 6975 (MEXU, XAL); al N de Tigrillos, G. CastilloCampos y M. E. Medina A. 28306 (XAL); estación Apazapan, camino a El Palmar, cerca al cerro, $M$. Cházaro B. y P. Hernández de Ch. 4269 (XAL). Municipio Atzalan, Hixtatahuía, ca. de la carretera Atzalan - Tlapacoyan, F. Ventura A. 97 (ENCB). Municipio Banderilla, along Xalapa - Perote hwy on NW side of Banderilla, M. Nee 25170 (XAL); principio del camino antiguo a Jilotepec, 


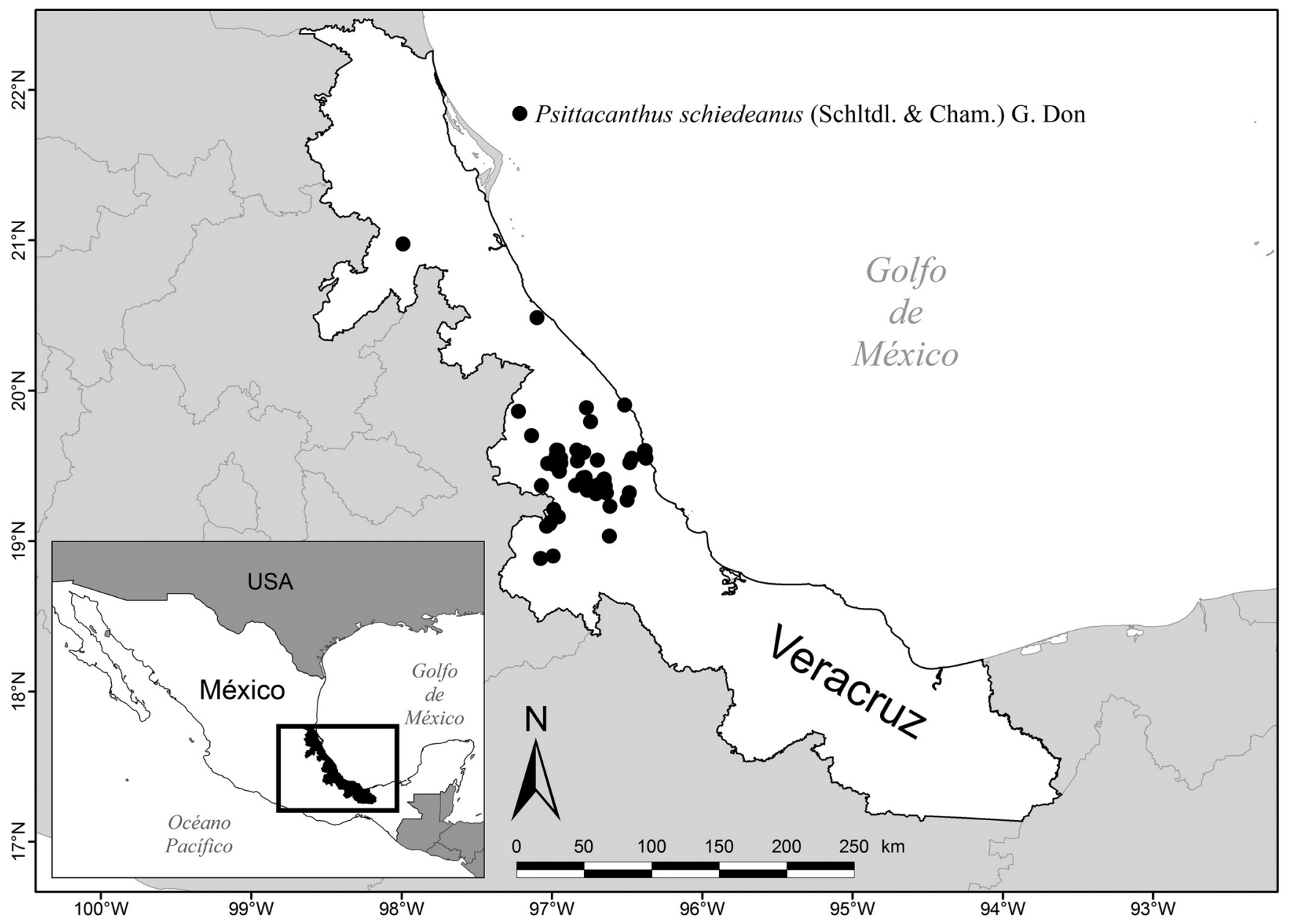

Figura 4: Distribución de Psittacanthus schiedeanus (Schltdl. \& Cham.) G. Don.

P. Padilla S. 59 (ENCB); rancho La Mesa, $2 \mathrm{~km}$ al NW de Banderilla, M. G. Zolá B. 506 (ENCB, XAL). Municipio Camarón de Tejeda, Villa Adalberto Tejeda, Concurso infantil popular s.n. (XAL). Municipio Chiconquiaco, cerro de Villa Rica, arriba de El Cedral, C. Gutiérrez B. 3571 (XAL). Municipio Chicontepec, La Pagua, $25 \mathrm{~km}$ SW de Tepetzintla, laderas de sierra de Otontepec, $G$. Castillo-Campos y A. Benavides M. 2330 (XAL). Municipio Coatepec, puente Nuevo, $4 \mathrm{~km} \mathrm{~W}$ de Xalapa, carretera antigua Xalapa - Coatepec, J. I. Calzada 1950 (ENCB, XAL); Pozo de Cantera, laderas del cerro de Chavarrillo, G. Castillo-Campos y J. L. Tapia M. 866 (ENCB, XAL); Cinco Palos, $5 \mathrm{~km}$ al NW, V. E. Luna M. 836 (XAL); La Pitaya, V. E. Luna M. 10306 (ENCB, MEXU). Muni- cipio Emiliano Zapata, cerro de Tepeapulco, camino de terracería Chavarrillo - El Palmar, J. I. Calzada y F. Vázquez B. 10241 (XAL); entre Tigrillos y Carrizal, sobre la carretera a la cementera, G. Castillo-Campos y M. E. Medina A. 28307 (XAL); along road from Baños Carrizal to Emiliano Zapata, 2-6 km SE of Emiliano Zapata, B. F. Hansen y M. Nee 7477 (XAL); carretera Xalapa - Veracruz, cerca de Plan del Río, R. Torres C. 09 (XAL); Plan del Río, F. Ventura A. 3011 (ENCB); La Cumbre, F. Ventura A. 4000 (ENCB). Municipio Fortín, Posada Loma, Fortín de Las Flores, L. I. Nevling y A. Gómez-Pompa 310 (XAL). Municipio Gutiérrez Zamora, $3 \mathrm{~km}$ al N de Gutiérrez Zamora, por la carretera a Boca de Lima, $G$. Castillo-Campos y M. E. Medina A. 28361 (XAL). Mu- 
nicipio Huatusco, $4 \mathrm{~km}$ camino a Coscomatepec, camino a Tenampa, S. Avendaño R. y J. I. Calzada 413 (XAL); puente Jamapa, camino Huatusco - Córdoba, W. Márquez R. 369 (MEXU, XAL); Huatusco, $1 \mathrm{~km}$ antes, a la derecha, B. Melo G. 25 (XAL); Coxcontla, F. Ventura A. 5681 (ENCB). Municipio Ixhuacán de los Reyes, después de Coyopolan, hacia Ixhuacán, M. Cházaro B. y P. Padilla S. 3724 (MEXU, XAL). Municipio Ixtaczoquitlán, Santa Ana Atzacan, N of Orizaba, M. Rosas R. 692 (XAL). Municipio Jalcomulco, barranca de Tlacuitlapa, entre Tuzamapan - Jalcomulco, camino Coatepec - Huatusco, G. Castillo-Campos y W. Bussey 3001 (ENCB, MEXU, XAL). Municipio Las Minas, Las Minas, camino a Puente Caballo, C. Durán E. y P. Burgos 753 (XAL). Municipio Naolinco, al S de Almolonga, G. Castillo-Campos et al. 18098 (MEXU, XAL); $2 \mathrm{~km}$ al S de Los Cafetales, $G$. Castillo-Campos et al. 19744 (MEXU, XAL); ca. de Almolonga, R. Ortega O. 1266 (XAL). Municipio Paso de Ovejas, barranca de Acazónica, 5 km al NW de Acazónica, M. E. Medina A. y F. Vázquez B. 329 (ENCB, MEXU, XAL); Mata Mateo, D. C. Nogueira T. 36 (XAL). Municipio Puente Nacional, Puente Nacional, A. Miranda J. s.n. (XAL). Municipio San Andrés Tlalnelhuayocan, represa de Xocollolapa, alrededor, San Andrés Tlalnelhuayocan, A. Flores-Palacios y J. G. García-Franco 918 (MEXU, XAL); rancho Mutmot, A. P. Vovides yard, A. P. Vovides 1509 (XAL); rancho Tejocotal, al E, P. Zamora C. 2754 (MEXU, XAL); Tlalnelhuayocan, orilla del río Pixquiac, P. Zamora C. 3008 (XAL); Tlalnelhuayocan, al SE, P. Zamora C. 3154 (MEXU, XAL). Municipio Tlaltetela, camino a Cueva del Abono, ejido Coetzala, L. Robles H. 313 (MEXU, XAL). Municipio Totutla, Totutla, $3 \mathrm{~km}$ adelante, camino a Huatusco, H. Oliva R. y M. Cházaro B. 103 (XAL). Municipio Uxpanapa, campamento Hnos. Cedillo, $1.3 \mathrm{~km}$, camino a La Laguna Xalapa, P. E. Valdivia Q. 530 (XAL). Municipio Xalapa, Xalapa, J. I. Calzada 2093 (MEXU, XAL); rancho Guadalupe, parque ecológico Francisco Javier Clavijero, km 2.5 del camino antiguo Xalapa - Coatepec, L. Monroy et al. 48 (ENCB, MEXU); vicinity of jardín botánico Clavijero, $4 \mathrm{~km} \mathrm{SW}$ of center of Xalapa, M. Nee 32847 (ENCB, MEXU); ca- ñada Tembladeras, P. Zamora C. 1778 (MEXU, XAL). Municipio Yecuatla, Chalahuite, camino a El Ojite, $C$. Gutiérrez B. 3736 (MEXU, XAL).

\section{Discusión}

Se han reportado más de 50 géneros de angiospermas y coníferas como hospederos del género Psittacanthus, incluyendo árboles, arbustos y monocotiledóneas arborescentes. Entre los principales hospederos se encuentran, entre otros, Acacia, Amphipterygium Schiede ex Standl., Annona L., Arbutus L., Baccharis L., Bursera, Casuarina L., Citrus, Coccoloba P. Browne, Crataegus L., Diphysa Jacq., Eucalyptus L'Hér., Ficus, Fraxinus L., Juglans L., Leucaena Benth., Liquidambar, Mimosa L., Nerium L., Olea L., Persea Mill., Pithecellobium Mart., Populus L., Prunus L., Prosopis L., Psidium L., Pseudosmodingium Engl., Pyrus L., Quercus L., Salix L., Spondias L. y Ulmus L. (Cházaro et al., 1992; García-Franco et al., 1995; Vázquez y Geils, 2002). Para Veracruz, las especies que tienen mayor diversidad de hospederos son P. schiedeanus y $P$. rhynchanthus, donde los más comunes son los géneros Acacia, Bursera, Ficus, Liquidambar, Quercus y Spondias. En Veracruz se observó que frecuentemente estas dos especies comparten hospederos principalmente del género Ficus y Bursera.

Entre las cuatro especies que habitan en Veracruz, Cházaro (2006) cita a Psittacanthus americanus (L.) Mart.; sin embargo, Kuijt (2009a) reconoce a P. americanus como especie exclusiva del Caribe y que los especímenes de la región continental identificados como ésta corresponden en su mayoría a $P$. rhynchanthus. También asevera que $P$. calyculatus es una especie extremadamente variable y en algunas ocasiones es difícil separarla de $P$. rhynchanthus y especialmente de $P$. schiedeanus. Sin embargo, con este trabajo se puede constatar que las tres especies pueden diferenciarse claramente.

Kuijt (2009a) señala la dificultad para separar las especies, comenta que $P$. rhynchanthus se diferencia de $P$. calyculatus y P. schiedeanus por presentar tallos cuadrangulares escasamente ramificados, flores color rojo, rectas, ápice del botón fuertemente curvado, eje articula- 
do color rojo, y crece en elevaciones bajas de la costa. Sin embargo, $P$. schiedeanus se puede diferenciar por su gran tamaño (Cuadro 1), ampliamente ramificado, flores color anaranjado con el ápice recto y más delgadas que en las otras dos especies (P. calyculatus y P. rhynchanthus) y el eje de la inflorescencia color verde y no articulado.

De acuerdo con lo reportado por Cházaro (2006), se deduce que de las cuatro especies presentes en el estado es relevante mencionar a $P$. ramiflorus que tiene preferencia geográfica en la vertiente del Pacífico (Chiapas, Guerrero, Jalisco, Nayarit, Oaxaca y Sinaloa) y sólo se registra de la vertiente del Golfo de México en Veracruz, hecho biogeográfico significativo tomando en cuenta el hábito parasítico de esta especie.

En cuanto a la especificidad por hospederos, $P$. calyculatus no presenta esta condición y puede parasitar una gran diversidad de árboles, incluyendo una situación particular, donde parasita a Beaucarnea gracilis Lem., caso raro de una monocotiledónea como hospedero de un muérdago. Por otra parte, P. ramiflorus sólo parasita árboles de Arbutus, Quercus y Matudaea Lundell (Cházaro, 2006). En las observaciones de campo se pudo ver que $P$. calyculatus tiene una distribución muy reducida en Veracruz y parasita árboles del género Acacia; la población local menciona que su presencia en la zona es muy reciente, no mayor a unos 20 años.

Se reconoce que Psittacanthus schiedeanus parasita más de 20 especies de árboles en los bosques de niebla del centro de Veracruz (Cházaro y Oliva, 1988), entre los que destacan como hospederos preferentemente Liquidambar styraciflua y Quercus germana Schltdl. \& Cham.; así también, Acacia pennatula (Schltdl. \& Cham.) Benth., Persea americana Mill., Platanus mexicana Moric. y Quercus leiophylla A. DC. (Cházaro y Oliva, 1988; López-de Buen y Ornelas, 1999, 2002; Cocoletzi et al., 2016), entre otros. Psittacanthus schiedeanus es una especie bastante agresiva para invadir hospederos y sus semillas logran germinar en ambientes poco apropiados para su establecimiento, como rocas y estructuras de cemento. Por esa razón es capaz de parasitar a Platanus mexicana que es una especie que cada año muda su cor- teza y con ello se desprende de epífitas y plantas parásitas de sus troncos.

En un estudio reciente (Cocoletzi et al., 2016) se ha determinado, a través de trabajos anatómicos, que Psittacanthus schiedeanus afecta de manera notoria a sus hospederos a nivel del parénquima. Así mismo, en Liquidambar styraciflua reduce gradualmente la estructura del floema que se encuentra cerca del haustorio. Sin embargo, en Quercus germana la estructura del floema se ve expandido en áreas parasitadas.

Por último, se ha mencionado que las especies del género Psittacanthus se usan en forma de té para el tratamiento de la presión arterial, específicamente $P$. calyculatus es utilizada como forrajera (Cházaro y Oliva, 1988) y algunos artesanos en Veracruz y Puebla la han empleado para elaborar artesanías de madera y bases de lámparas (Cházaro, 1995).

\section{CONTRIBUCIÓN DE AUTORES}

GCC diseñó el estudio, participó en el muestreo de campo, revisión de especímenes y en la integración y redacción del manuscrito final. MEMA colaboró en el muestreo de campo, elaboración de mapas, y revisión de especímenes y del manuscrito final. RAR contribuyó en la redacción de la introducción, la discusión y revisión del manuscrito final.

\section{FINANCIAMIENTO}

Este estudio fue apoyado con los recursos fiscales del Instituto de Ecología, A.C. (GCC 20030-10134).

\section{AgRAdECIMIENTOS}

Se agradece a los curadores de los herbarios ENCB, MEXU, XAL por permitirnos revisar las colecciones depositadas en sus acervos. A Ma. del Rosario Landgrave Ramírez por la edición de los mapas, y a Jesús Pale Pale por la integración de las fotografías.

\section{LITERATURA CITADA}

Cházaro B., M. J. 1995. Los muérdagos (Loranthaceae) del estado de Jalisco. In: Cházaro B., M. J., M. Lomelí, R. Acevedo R. y S. Ellerbracke R. (comps.). Antología 
botánica del estado de Jalisco. Universidad de Guadalajara. Guadalajara, México. Pp. 74-77.

Cházaro B., M. J. 2006. Las plantas parásitas de México: Florística y Fitogeografía. Tesis de doctorado. Centro Universitario de Ciencias Biológicas y Agropecuarias, Universidad de Guadalajara. Guadalajara, México. 172 pp.

Cházaro B., M. J. y H. Oliva. 1988. Loranthaceae del centro de Veracruz y zona limítrofe de Puebla. Parte 5. Cactáceas y Suculentas Mexicanas 33(3): 71-75.

Cházaro B., M. J., F. M. Huerta M., R. M. Patiño B., R. Sánchez F., E. Lomelí M. y A. Flores M. 1992. Los muérdagos (Loranthaceae) de Jalisco, parásitas poco conocidas. Ciencia y Desarrollo 17(102): 70-85.

Cocoletzi, E., G. Ángeles, G. Ceccantini, A. Patrón y J. F. Ornelas. 2016. Bidirectional anatomical effects in a mistletoe-host relationship: Psittacanthus schiedeanus mistletoe and its hosts Liquidambar styraciflua and Quercus germana. American Journal of Botany 103(6): 986-997. DOI: https://dx.doi.org/10.3732/ajb.1600166

ESRI. 2014. ArcGIS, versión 10.2.2. Environmental Systems Research Institute. Redlands, USA.

García-Franco, J. G., V. Rico-Gray y M. Palacios-Rios. 1995. Parasitismo de Psittacanthus calyculatus (Loranthaceae) sobre Beaucarnea gracilis (Nolinaceae) en el Valle de Zapotitlán de las Salinas, Puebla, México. Cactáceas y Suculentas Mexicanas 40: 62-65.

Geils, B. W. y I. Vázquez C. 2002. Loranthaceae and Viscaceae in North America. In: Geils, B. W., J. C. Tovar y B. Moody (coords.). Mistletoes of North American conifers. Chapter 1. General Technical Report. Department of Agriculture. Ogden, USA. Pp. 1-8.

IUCN. 2017. The International Union for Conservation of Nature. Red List of Threatened Species. Version 20172: http://www.iucnredlist.org/ (consultado septiembre de 2017).
JSTOR. 2017. JSTOR Global Plants: https://plants.jstor.org/ collection/TYPSPE (consultado septiembre de 2017).

Kuijt, J. 2009a. Monograph of Psittacanthus (Loranthaceae). Systematic botany monographs. Vol. 86. American society of plant taxonomists. Ann Harbor, USA. 361 pp.

Kuijt, J. 2009b. 58. Loranthaceae. Flora Mesoamericana 2(1): $1-15$.

López-de Buen, L. y J. F. Ornelas. 1999. Frugivorous birds, host selection and the mistletoe Psittacanthus schiedeanus, in central Veracruz, México. Journal of Tropical Ecology 15(3): 329-340. DOI: https://dx.doi.org/10.1017/ S0266467499000851

López-de Buen, L. y J. F. Ornelas. 2002. Host compatibility of the cloud forest mistletoe Psittacanthus shiedeanus (Loranthaceae) in central Veracruz, Mexico. American Journal of Botany 89(1): 95-102. DOI: https://dx.doi. org/10.3732/ajb.89.1.95

SEMARNAT. 2010. Norma Oficial Mexicana NOM-059SEMARNAT-2010, Protección ambiental-Especies nativas de México de flora y fauna silvestres-Categorías de riesgo y especificaciones para su inclusión, exclusión o cambio-Lista de especies en riesgo. Secretaría del Medio Ambiente y Recursos Naturales. Diario Oficial de la Federación. Cd. Mx., México. http://dof.gob.mx/nota_ detalle.php?codigo $=5173091 \&$ fecha $=30 / 12 / 2010$.

Standley, P. C. y J. A. Steyermark. 1946. Loranthaceae. Flora of Guatemala 24(4): 62-86.

Toledo, C. 1960. Loranthaceae. Flora of Panama 47(4): 263359.

TROPICOS. 2017. Tropicos.org. Missouri Botanical Garden. http://www.tropicos.org (consultado septiembre de 2017).

Vázquez C., I. y B. W. Geils. 2002. Psittacanthus in Mexico. In: Geils, B. W., J. C. Tovar y B. Moody (coords.). Mistletoes of North American conifers. Chapter 2. General Technical Report. Department of Agriculture. Ogden, USA. Pp. 9-18. 